\title{
Chinese Subject-Relative Clauses Are Easier to Comprehend Than the Object-Relative Clauses in Natural Situations*
}

\author{
HE Wen-guang \\ School of Education, Qufu Normal Uinversity, Qufu, China
}

\begin{abstract}
Studies on Indo-European languages argue that subject relative clauses (SRCs) are easier to process than object relative clauses (ORCs), and the processing preference for SRCs was manipulated by the animacy of the noun phrases (NPs) in the internal-clause and in the main clause. This paper aims at investigating the difficulty in Chinese RCs processing by subjective ratings and the results showed: (1) As European languages, Chinese RCs processing also shows a preference for SRCs, which was consistent with experience-based account, as well as NPAH in part. (2) A strong animacy effect from the head noun phrases (NPs) was found during Chinese RCs processing. In all, compared with other accounts, experience-based account offers more reasonable explanations about Chinese RCs comprehension in natural situations.
\end{abstract}

Keywords: relative clause, animacy effect, Chinese

\section{Introduction}

Relative clauses (RCs) are one type of wh-constructions where relativizers such as "who" or "which" appear in designated positions, leaving a gap or trace (marked as $t$ in the example sentences below) in their original position. Relativizers such as "who" or "that" are traditionally called fillers, since they can be mapped to fill gaps that are posited in the canonical position for noun phrases. RCs processing has been an important topic for half a century owning to its grammatical complexity as well as its rich typology (Gibson, 1998). Psycholinguistic examinations of RCs have provided many insights on the architecture and mechanisms of human language comprehension. Accordingly, RC construction offers a window of opportunities for studying the cognitive mechanism under production and comprehension (Hawkins, 2004). Although RCs differ considerably in their syntactic structures across languages, subject-extracted relative clauses as (1a) and object-extracted relative clauses as (1b) are more popular and typical. Thus, investigations about the processing of the two types of RCs have being the hottest issues in past decades. In the subject-extracted relative clauses (SRCs), the reporter serves as the doer of the action "attacked". By contrast, in the object-extracted relative clauses (ORCs), the reporter is the object of the action "attacked". These two types of RCs are distinguished by the role that the head noun (i.e., the reporter) plays in the relative clause.

1a Subject-extracted relative clause (SRC):

The reporter[filler] who $[t]$ attacked the senator admitted the error.

\footnotetext{
${ }^{*}$ Acknowledgements: This research was financially supported by the National Social Science Foundation of China (Grant NO. 13CYY026).

HE Wen-guang, Ph.D., School of Education, Qufu Normal University.
} 
1b Object-extracted relative clause (ORC):

The reporter[filler] who the senator attacked $[t]$ admitted the error.

In the past decades, SRCs or ORCs processing has been thoroughly investigated in different languages with various methods such as self-paced reading (Gibson, Desmet, Grodner, Watson, \& Ko, 2005), eye-movement tracking (Traxler, Morris, \& Seely, 2002), event-related potentials (ERPs) (Müller, King, \& Kutas, 1997) and functional magnetic resonance imaging (fMRI) (Caplan, Stanczak, \& Waters, 2008). A well-established result of such studies in Indo-European languages is that SRCs are easier to comprehend than ORCs. This has been shown to be the case in English (Caplan, Stanczak, \& Waters, 2008), in German (Schwartz, 2007), in Italian (Domenica \& Matteo, 2009), in Dutch (Mak, Vonk, \& Schriefers, 2006) and in French (Cohen \& Mehler, 1996).

Of all the theoretical frameworks developed for explicating the difference in difficulty between SRCs and ORCs, two accounts were worthy for us to talk about below.

Experience-based account

According to language experience-based accounts, the observed difference between processing of object and subject relative clauses may be explained by differences in exposure to statistical regularities of language (MacDonald \& Christiansen, 2002). The more frequent the words, phrases, and structures are, the more easily they can be activated and retrieved. The processing asymmetry between SRCs and ORCs more likely arises from their distribution difference in natural languages.

Noun Phrase Accessibility Hierarchy (NPAH)account

The NPAH is grounded on the observation that there are more languages which can relativize on subject than languages which can also relativize on direct object, on direct object than also on indirect object, on indirect object than also on oblique, and so forth. This suggests strongly that "the further we descend the NPAH, the harder it is to relativize” (Keenan \& Comrie, 1977). NPAH puts the subject highest in the hierarchy for all languages, so this account predicts that subject-extracted RCs should be universally easier than object-extracted RCs, regardless of other properties shown in different languages.

Chinese also has RC structures. However, unlike RCs in Indo-European languages, Chinese RCs as (2) have its special characters.

2a Subject-modifying SRC (S-SRC):

[Gap 攻击议员的]律师 Filler不喜欢那位政客。

attacked senator (de) lawyer doesn't like that politician

The lawyer Filler that_Gap attacked the senator doesn't like the politician.

2b Subject-modifying ORC (S-ORC):

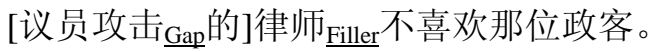

senator attacked (de) lawyer doesn't like that politician

The lawyer Filler $_{\text {that }}$ the senator attacked ${ }_{\text {Gap }}$ doesn't like the politician.

First, English RCs are head-initial sentences with the filler before the gap, while Chinese RCs are head-final sentences with the gap before the filler, which was challenging to RCs processing theory based on filler-gap dependency.

Second, Chinese RCs have no definite relational markers as "that" and "who" in English. In Chinese, 的 (de) serves as the relative clause marker in the sentence, but its function is not same as the word "that" or "who" in English. 
Owing to these specific properties, Chinese RCs have been regarded as conducive materials for retesting models of sentence comprehension. There have been several recent studies conducted on processing of Chinese RCs, which have yielded conflicting results.

In sum, Chinese RCs processing is becoming a hot issue in current for their typologically properties, but most studies focused on the processing difficulty of Chinese RCs with double animate NPs in the internal-clause and the main clause, and studies focusing on the processing of Chinese RCs with different animacy configuration in the internal-clause and the main clause were scare, which was a limit to generalize findings from Chinese RCs processing, for studies based on corpus analysis found that RCs with different animacy configuration occur more frequently than RCs with double animate NPs (Wu, 2009). Specifically, RCs with different animacy configuration are more frequent and typical than RCs with double animate NPs. We aim at exploring the following questions regarding Chinese RCs processing in this paper:

(1) Is there processing asymmetry on RCs in Chinese as like in English, Dutch or German?

(2) What accounts for the processing asymmetry between subject and object relatives in Chinese?

\section{Method}

\section{Materials}

Eighty sets of SRCs and ORCs were constructed. Forty sets of RCs have double animate NPs in the internal-clause and in the main clause ( $3 a \& 3 b$ ), the other forty sets of RCs have NPs from different categories, some are animate entities as 记者 (reporter), and some are inanimate entities as 新闻 (news). Of them, twenty sets of RCs have head animate NPs in the main clause and inanimate NPs in the internal-clause (4a \& $4 b$ ), and the other twenty sets of RCs have inanimate head NPs in the main clause and animate NPs in internal-clause ( $5 a \& 5 b$ ). The sentence followed a rigorous subject-verb-object word order so that the verb was always adjacent to its major noun phrase argument. The ORCs were derived from the corresponding SRCs by exchanging the first word and the second word, so SRCs and ORCs were same at the level of word.

3a Chinese subject relative clause (SRC: double animacy configuration)

攻击律师的议员呼吁民众保持冷静。

The senator that attacked the lawyer asked people to keep calm.

3b Chinese object relative clause (ORC: double animacy configuration)

律师攻击的议员呼吁民众保持冷静。

The senator that the lawyer attacked asked people to keep calm.

4a Chinese subject relative clause (SRC: inanimate-animate configuration) 曝光新闻的记者引起政府的关注。

The reporter that exposed the news attracted the government's attention.

$4 \mathrm{~b}$ Chinese object relative clause (ORC: inanimate-animate configuration)

新闻曝光的记者引起政府的关注。

The reporter that the news exposed attracted the government's attention.

5a Chinese subject relative clause (SRC: animate-inanimate configuration)

曝光记者的新闻引起政府的关注。

The news that exposed the reporter attracted the government's attention.

5b Chinese object relative clause (ORC: animate-inanimate configuration)

记者曝光的新闻引起政府的关注。 
The news that the reporter exposed attracted the government's attention.

The plausibility of the materials

One hundred and sixty participants rated the plausibility of these sentences on a scale of 1 (more natural) to 5 (more unnatural). They were asked to judge the naturalness of the events described in the sentences in the real world, that is, how likely they were to occur, and the results were shown in Table 1. T-t tests showed there was no significant difference between SRCs and ORCs.

Table 1

The Plausibility Results of RCs Across All Conditions ( $M \pm S D)$

\begin{tabular}{lllll}
\hline & $\mathrm{M}$ & $\mathrm{SD}$ & $\mathrm{t}$ & $\mathrm{p}$ \\
\hline $\mathrm{SRC}_{3 \mathrm{a}}$ & 1.61 & 0.32 & -0.94 & 0.35 \\
$\mathrm{ORC}_{3 \mathrm{~b}}$ & 1.66 & 0.42 & -1.01 & \\
$\mathrm{SRC}_{4 \mathrm{a}}$ & 1.81 & 0.33 & & 0.32 \\
$\mathrm{ORC}_{4 \mathrm{~b}}$ & 1.84 & 0.37 & -1.29 & \\
$\mathrm{SRC}_{5 \mathrm{a}}$ & 1.67 & 0.32 & 0.21 \\
ORC $_{5 \mathrm{~b}}$ & 1.71 & 0.38 & & \\
\hline
\end{tabular}

\section{Participants}

Four hundred and eighty college students from Beijing Normal University participated in the survey. Their age ranged from 17 to 23, and they were all native Chinese speakers with normal or corrected-to-normal vision.

\section{Procedure}

In order to avoid the fatigue effect and other interference, materials were divided into two parts (part 1 \& part 2), each part including eighty items and eighty fillers. Each participant reads only one part of the two.

After some explanations about the survey, participants were asked to rate the processing difficulty of these sentences on a scale of 1 (more easily to comprehend) to 7 (more difficult to comprehend) according to their estimates. All the investigations were done in a quiet classroom, the entire testing lasted approximately 30 minutes. Participants were paid for their attendance.

\section{Results and Discussion}

Comparisons between SRCs and ORCs across all conditions and the results are shown in Table 2.

Table 2

The Rating Results of RCs Processing in All Conditions ( $M \pm S D$ )

\begin{tabular}{lllllllll}
\hline & $\mathrm{SRC}_{3 \mathrm{a}}$ & $\mathrm{ORC}_{3 \mathrm{~b}}$ & $\mathrm{SRC}_{4 \mathrm{a}}$ & $\mathrm{ORC}_{4 \mathrm{~b}}$ & $\mathrm{SRC}_{5 \mathrm{a}}$ & $\mathrm{ORC}_{5 \mathrm{~b}}$ & $\mathrm{SRC}_{4 \mathrm{a}+5 \mathrm{a}}$ & $\mathrm{ORC}_{4 \mathrm{~b}+5 \mathrm{~b}}$ \\
\hline $\mathrm{M}$ & 2.43 & 2.78 & 2.17 & 2.28 & 2.25 & 2.27 & 2.21 & 2.28 \\
$\mathrm{SD}$ & 0.58 & 0.66 & 0.46 & 0.58 & 0.58 & 0.62 & 0.45 & 0.56 \\
\hline
\end{tabular}

\section{The Difference in Difficulty Between SRCs and ORCs With Double Animacy Configuration}

In this paper, we found Chinese SRCs were easier to comprehend than ORCs, F $(1,238)=247.84, M S E=$ 14.02, $p<.0001$. Our results confirmed with predictions from NPAH and accounts based on experience. According to NPAH, subject is the easiest to be relativized than other grammatical relations, which means SRCs should be universally easier than ORCs. On the other hand, language experience was an important factor in sentence comprehension, as tuning accounts claimed that processing preferences are driven by the frequency 
of occurrence of particular sentence structures (Mitchell, Cuetos, Corley, \& Brysbaert, 1995). To explore factors affecting RCs processing, Hisao and Gibson (2003) analyzed Chinese RCs distribution based on the Chinese Treebank, version 3.0, a corpus consisting of approximately 100,000 words that were taken from the Xinhua newswire between 1994 and 1998, which published by the Linguistic Data Consortium. They found 882 instances of RCs in total, 375 (42.5\%) of which were ORCs and the remaining 507 (57.5\%) instances are SRCs.

\section{The Difference in Difficulty Among RCs With Animate-Inanimate Configuration and Reverse}

One two-factor ANOVAS was performed and the results showed that the main effect of RCs types was significant, $\mathrm{F}(1,239)=8.84, M S E=1.05, p<.01$, and the main effect of animacy configuration was not significan, $\mathrm{F}(1,239)=2.10, M S E=0.31, p=0.15$, but there was a significant interaction between RCs type and animacy configuration, $\mathrm{F}(1,239)=3.82, M S E=0.36, p=.05$. A simple analysis showed that SRCs were easier to comprehend than ORCs in the conditions with inanimate-animate configuration (e.g., $4 a \& 4 b)$, F (1, 239) $=4.57, M S E=0.67, p<.05$, and no difference was found between SRCs and ORCs in the conditions with animate-inanimate configuration (e.g., $5 a \& 5 b)$, F $(1,239)=0.01, M S E=.00, p=0.91$.

Specifically, SRCs were easier difficult than ORCs in the conditions with inanimate-animate configuration (e.g., $4 a \& 4 b$ ), but no difference in the conditions with animate-inanimate configuration (e.g., $5 a \& 5 b$ ), which was also confirmed with experience-based account. Wu (2009) made a further analysis about Chinese RCs distribution according to the aniamcy configuration of the head NPs in the internal-clause and in the main clause based on the Chinese Treebank, version 5.0, a revised corpus based on version 3.0. Results showed that SRCs were more frequent than ORCs in the conditions with inanimate-animate configuration $(76.34 \%$ vs. 23.66\%), while in the conditions with animate-inanimate configuration, ORCs were same as SRCs in frequency (51.39 \% vs. 49.61\%). Additionally, our results were not confirmed with the NPAH and the canonical accounts.

\section{The Animacy Effect in Chinese RCs Processing}

Studies have showed that the animacy of the head NPs have an important role in RCs processing (Mak, Vonk, \& Schriefers, 2006). For example, some studies found SRCs were easier to comprehend than ORCs when the NPs in the internal-clauses and in the main clause were both animate entities as (6), but when the head NPs were from different categories as (7), the difference in difficulty between SRCs and ORCs disappeared.

6a The lawyer that irritated the banker filed a hefty lawsuit. (SRC)

$6 \mathrm{~b}$ The lawyer that the banker irritated filed a hefty lawsuit. (ORC)

7a The movie that pleased the director received a prize at the film festival. (SRC)

7b The movie that the director watched received a prize at the film festival. (ORC)

We also found this animacy effect in Chinese comprehension by off-line paradigm. As discussed in section 3.2, this effect may be from language experience. But some scholars attributed the animacy effect to similarity-interference. According to Gordon, Hendrick and Johnson (2001), the difficulty readers have with ORCs as in (8) comes from the fact that these RCs require two NPs to be stored in memory and subsequently accessed whereas in English this is not the case for SRCs. The difficulty of accessing the two representations is reduced when they are dissimilar, as in (9).

(8) The banker that the lawyer praised climbed the mountain.

(9) The banker that you praised climbed the mountain. 
To explore whether the explanation of the difference in processing difficulty between SRCs and ORCs in terms of NPs to be stored in memory holds for Chinese RCs or not, one two-factor ANOVAS was performed again in the conditions with double animacy configuration and different animacy configuration in the internal-clause and in the main clause (e.g., SRC3a/ORC3b vs. SRC4a+5a/ORC4b+5b). The main effect of animacy configuration was significant, $\mathrm{F}(1,238)=149.39, \mathrm{MSE}=10.03, p<.0001$, which shows that RCs in conditions with double animate entities in the internal-clause and in the main clause were more difficult to comprehend than RCs in conditions with different animate entities. The main effect of clause types was significant, $\mathrm{F}(1,238)=312.81, M S E=31.52, p<.0001$, and the interaction between animacy configuration and clause types was also significant, $\mathrm{F}(1,238)=92.9, M S E=4.53, p<.0001$, simple analysis shows that ORCs were more difficult than SRCs in conditions with different animacy configuration in the internal-clause and in the main clause, and in conditions with double animacy configuration, ORCs were much more difficult to comprehend than SRCs.

Putting findings in section 3.1, 3.2 and 3.3 together, we have good reasons to assume that the difference in processing difficulty between SRCs and ORCs and the animacy effect in RCs comprehension were mainly from language experience.

\section{Conclusion}

This paper aims to investigate Chinese RCs processing through participants' subjective assessment in natural reading context by off-line paradigm and results showed that Chinese SRCs are easier to comprehend than Chinese ORCs in conditions with double animate entities in the internal-clause and in the main clause, as well as in conditions with inanimate-animate configuration, but in conditions with animate-inanimate configuration, no differences were found between SRCs and ORCs. What's more, aniamcy effect was also found in Chinese RCs comprehension as in English. Our results confirmed with experience-based accounts, as well as NPAH in part.

\section{References}

Caplan, D., Stanczak, L., \& Waters, G. (2008). Syntactic and thematic constraint effects on blood oxygenation level dependent signal correlates of comprehension of relative clauses. Journal of Cognitive Neuroscience, 20, 643-656.

Cohen, I., \& Mehler, J. (1996). Clicking monitoring revisited: An on-line study of sentence comprehension. Memory \& Cogniton, 24, 94-102.

Domenico, A., \& Matteo, R. D. (2009). Processing Italian relative clauses: Working memory span and word order effects on RTs. The Journal of General Psychology, 136, 387-406.

Gibson, E. (1998). Linguistic complexity: Locality of syntactic dependencies, Cognition, 68, 1-76.

Gibson, E., Desmet, T., Grodner, D., Watson, D, \& Ko, K. (2005). Reading relative clauses in English. Cognitive Linguistics, 16, 313-354.

Gordon, P. C., Hendrick, R., \& Johnson, M. (2001). Memory interference during language processing. Journal of Experimental Psychology: Learning, Memory, and Cognition, 27, 1411-1423.

Hawkins, J. (2004). Efficiency and complexity in grammar. Oxford: Oxford University Press.

Hsiao, F., \& Gibson, E. (2003). Processing relative clauses in Chinese. Cognition, 90, 3-27.

Keenan, K., \& Comrie, B. (1977). Noun phrase accessibility and universal grammar. Linguistic Inquiry, 8, 63-99.

MacDonald, M. C., \& Christiansen, M. H. (2002). Reassessing working memory: Comment on Just and Carpenter (1992) and Waters and Caplan (1996). Psychological Review, 109, 35-54.

Mak, W. M., Vonk, W., \& Schriefers, H. (2006). Animacy in processing relative clauses: The hikers that rocks crush. Journal of Memory and Language, 54, 466-490. 
Mitchell, D. C., Cuetos, F., Corley, M. M. B., \& Brysbaert, M. (1995). Exposure-based models of human parsing: Evidence for the use of coarse-grained (nonlexical) statistical records. Journal of Psycholinguistic Research, 24, 469-488.

Müller, H., King, J. K., \& Kutas, M. (1997). Event-related potentials elicited by spoken relative clauses. Cognition Brain Research, 5, 193-203.

Schwartz, F. (2007). Processing presupposed content. Journal of Semantics, 24, 373-416.

Traxler, M. J., Morris, R. K., \& Seely, R. E. (2002). Processing subject and object relative clauses: Evidence from eye movements. Journal of Memory and Language, 47, 69-90.

Wu, F. F. (2009). Factors affecting relative clause processing in Mandarin (The doctoral dissertation of University of Southern California). 